\title{
In situ Diagnosis of Helicobacter pylori Infection Using the Endoscopic Kyoto Scoring System
}

\author{
Eunsun Lim, Ik Hyun Jo, Yeon-Ji Kim, Woo Chul Chung \\ Department of Internal Medicine, St. Vincent's Hospital, College of Medicine, The Catholic University of Korea, Suwon, Korea
}

\begin{abstract}
Background/Aims: This study aimed to investigate the possibility of in situ diagnosis of Helicobacter pylori (H. pylori) infection during endoscopic examination. The predictive infection value was estimated using the endoscopic Kyoto scoring system (EKSS), and specific endoscopic findings were evaluated for diagnosing $H$. pylori infection in $H$. pylori naïve patients and those with a eradication history.

Materials and Methods: A total of 836 patients with $H$. pylori infection were analyzed. The state of the infection was predicted using the EKSS and specific endoscopic findings.

Results: Patients were classified into two groups: the $H$. pylori naïve group and the group with a the bacterial eradication history The area under the curve (AUC) on receiver operating characteristics analysis was 0.90 for EKSS in H. pylori naïve patients and 0.83 for the other group patients. For patients with open type atrophy and/or intestinal metaplasia, EKSS $(24.4 \%$; $95 \%$ CI, $12.4 \sim 0.3 \%)$ and regular arrangement of collecting venules (RAC) (46.3\%; 95\% CI, 30.7 62.9\%) showed low specificities. Mucosal swelling (66.2\%; 95\% CI, 62.5 69.7\%) and sticky mucus (80.5\%; 95\% CI, 74.8 85.2\%) presented relatively high positive predictive values for $H$. pylori infection in naïve patients, whereas reflux esophagitis, hematin, red streak, and duodenitis exhibited high negative predictive values in patients with a $\mathrm{H}$. pylori eradication history $(98.0 \%$; 95\% CI, 96.4 99.1\%).

Conclusions: EKSS and RAC are excellent tools for predicting $H$. pylori infection. However, they have a limited role in patients with open type atrophy and/or intestinal metaplasia. Specific endoscopic findings could help predict the infection state. (Korean J Helicobacter Up Gastrointest Res 2021;21:322-332)
\end{abstract}

Key Words: Diagnosis; Endoscopy; Helicobacter pylori

\section{INTRODUCTION}

Helicobacter pylori (H. pylori) infection produces a prolonged inflammation of gastric mucosa. It plays a causative role in peptic ulcer disease, gastric adenocarcinoma, and gastric mucosa-associated lymphoid tissue lymphoma. ${ }^{1-3}$ Gastric adenocarcinoma can develop from chronic atrophy, intestinal metaplasia, and dysplasia due to a prolonged inflammation caused by $H$. pylori infection. ${ }^{4,5}$ Therefore, eradication of $H$. pylori infection is important for primary prevention of gastric carcinogenesis.

Nevertheless, gastric adenocarcinoma has been reported in a substantial number of patients after $H$. pylori eradication. It is called ' $H$. pylori negative gastric adenocarcinoma'. It

Received: September 6, 2021 Revised: October 9, 2021 Accepted: November 3, 2021 Corresponding author: Woo Chul Chung

Department of Internal Medicine, St. Vincent's Hospital, College of Medicine, The Catholic University of Korea, 93 Jungbu-daero, Paldal-gu, Suwon 16247, Korea Tel: +82-31-249-7138, Fax: +82-31-253-8898, E-mail: jwchulkr@ catholic.ac.kr Our study is supported by a research grant from the Korea United Pharmaceutical Co., Seoul, Korea. has been hypothesized that precancerous lesions (atrophy or intestinal metaplasia) might have potential autonomy for gastric carcinogenesis. ${ }^{6}$ There might be an irreversible step at certain time of gastric carcinogenesis. It is believed there is a point of no return. ${ }^{7}$ To overcome the autonomy of gastric carcinogenesis, early detection with subsequent eradication of $H$. pylori before severe atrophy and intestinal metaplasia could be the best cost-effective strategy to prevent gastric adenocarcinomas. In situ diagnosis of $H$. pylori infection on endoscopic examination can contribute to its prevention strategy. When it is combined with PCR test for antibiotic resistance genes of $H$. pylori strains on endoscopic examination in situ, therapeutic improvement can be achieved based on information of antibiotic resistance. ${ }^{8}$

Previously, for discriminating H. pylori infection, several endoscopic findings have been emphasized, including the presence of atrophy, intestinal metaplasia, diffuse redness, spotty redness, mucosal swelling, enlarged folds, sticky mucus, nodularity, hyperplastic polyp, xanthoma, and de-

Copyright $\odot 2021$ Korean College of Helicobacter and Upper Gastrointestinal Research

(a) The Korean Journal of Helicobacter and Upper Gastrointestinal Research is an Open-Access Journal. All articles are distributed under the terms of the Creative Commons Attribution Non-Commercial License (http:// creativecommons.org/licenses/by-nc/4.0) which permits unrestricted non-commercial use, distribution, and reproduction in any medium, provided the original work is properly cited. 
pressed erosion known to have positive correlations with H. pylori infection, whereas regular arrangement of collecting venules (RAC), fundic gland polyp, red streaks, raised erosion, hematin, patchy redness, and map-like redness are negatively correlated with $H$. pylori infection. ${ }^{9-12}$ Several endoscopic features have low response rates for endoscopists. For example, fundic gland polyp and map like redness have low response rates due to their rarity, although these are closely related to $H$. pylori negative infection. ${ }^{9,10}$

In 2015, a global consensus meeting was held at Kyoto, Japan to develop consensus on classification of chronic gastritis, appropriate diagnostic assessment of gastritis, and recommended treatment for $H$. pylori gastritis. ${ }^{13}$ Endoscopic Kyoto scoring system (EKSS) was also proposed, in that meeting. The presence of atrophy, intestinal metaplasia, enlarged folds, nodularity, and diffuse redness were adapted for EKSS, which was designed for the detection of $H$. pylori infection and risk estimation of gastric cancer. ${ }^{14,15}$ Especially, diffuse redness based on the degree of visibility of RAC at body revealed by imaging enhanced endoscopy could be a useful discriminative tool for the detection of $H$. pylori infection. Although EKSS was a good predictor of $H$. pylori infection with high accuracy in a previous report, ${ }^{16}$ there were several limitations to acknowledge the usefulness of EKSS. First of all, its cutoff value was ambiguous. When patients had a past history of $H$. pylori eradication therapy, its useful was questionable. $^{17}$

The aim of this study was to evaluate the diagnostic predictive value of using EKSS. This study enrolled $H$. $p y^{-}$ lori naïve patients and those with a history of $H$. pylori eradication. Whether other specific endoscopic findings (mucosal swelling, sticky mucus, red streak, and hematin) could be useful for the diagnosis of $H$. pylori infection was determined. We also evaluated whether endoscopic duodenitis and reflex esophagitis including minor changes were correlated with $H$. pylori infection state.

\section{MATERIALS AND METHODS}

\section{Participants}

This clinical trial was carried out between May 2019 and May 2020. This study was approved by the Institutional Review Board of St. Vincent's Hospital, The Catholic University of Korea (approval no. VC19OESI0103). They also approved a consent procedure. We prospectively enrolled patients who were referred to the gastroenterology unit for evaluation at St. Vincent's Hospital, college of Medicine, The Catholic University of Korea. Patients were eligible for recruitment if they were older than 18 years of age. Exclusion criteria were: 1) active gastric and duodenal ulcers that might influence the estimation of $H$. pylori infection state, 2) history of gastrectomy, 3) use of proton pump inhibitor and histamine receptor-2 blocker within 2 weeks, or 4) treatment with antibiotics within 4 weeks. Patients were also excluded if they had significant renal, hepatic, cardiovascular, metabolic, or hematological disorders.

\section{Diagnosis of $H$. pylori infection}

\section{1) H. pylori naïve patients}

The diagnosis of $H$. pylori infection was made based on two or more positive results of rapid urease test (RUT), PCR, urea breath test (UBT), histologic evidence by silver stain, and serologic test. Two biopsies were used for RUT (CLO ${ }^{\circledR}$ test; Kimberly-Clark, Irving, TX, USA). DNAs were extracted from specimens using QIAamp DNA Mini kit (Qiagen, Valencia, CA, USA) according to the manufacturer's protocol and used for PCR. DNAs were stored at $-20^{\circ} \mathrm{C}$ until use. PCR (Seeplex ${ }^{\circledR}$ ClaR-H. pylori ACE Detection; Seegene Institute of Life Science, Seoul, Korea) was performed to identify point mutation-containing gene fragments according to the manufacturer's recommendations. ${ }^{13} \mathrm{C}$-urea breath test was performed with a pranactin-citric drug product, a component of a BreathTek UBT Kit (Korea Otsuka Pharmaceutical Co. Ltd., Seoul, Korea). Three grams of reconstituted pranactin-citric containing $75 \mathrm{mg}$ of ${ }^{13} \mathrm{C}$-urea was ingested by the patient. Breath samples before and 20 minutes after the administration of ${ }^{13} \mathrm{C}$-urea were collected after a mouthwash. ${ }^{13} \mathrm{C} /{ }^{12} \mathrm{C}$ ratios in breath samples were measured 
with an infrared spectrophotometer (UBiT-IR300; Korea Otsuka Pharmaceutical Co. Ltd.). Changes in ${ }^{13} \mathrm{C}$ value over baseline were expressed as $\Delta^{13} \mathrm{C}$. A positive result was defined as an increase of $>2.5 \%$. The presence of serum IgG antibodies against H. pylori (Enzygnost ${ }^{\circledR}$, Dade Behring, Marburg, Germany) was determiend. Antibody titer $>15$ $\mathrm{U} / \mathrm{mL}$ was classified as $H$. pylori sero-positive, whereas a titer $<10 \mathrm{U} / \mathrm{mL}$ was considered as $H$. pylori sero-negative following the manufacturer's instructions. $H$. pylori-negative patients in this study were judged as $H$. pylori-uninfected patients if two or more tests showed negative results.

2) H. pylori negative patients

The diagnosis of negative infection state of $H$. pylori was made based on two or more negative results of RUT, PCR, UBT, histologic evidence by silver stain, and serologic test.

3) Patients with a history of $H$. pylori eradication

Confirmation of successful eradication by UBT was obtained from medical records. For patients with a history of successful eradication, the diagnosis of $H$. pylori re-infection was made based on one or more positive results of RUT, PCR, UBT, and histologic evidence by silver stain. The patients with positive result of serum antibody test only was excluded in this study.

\section{Evaluation of endoscopy}

To reduce inter-observer variation, physicians with varying endoscopy experience were invited to educate the EKSS for $H$. pylori infection. Esophagoduodenoscopy was performed using a GIF-XQ 260 (Olympus Medical, Inc.
Tokyo, Japan). Endoscopic examinations were performed by one endoscopist (Chung WC), an actively practicing gastroenterologist with experience of $>500$ diagnostic endoscopies per year for 20 years. He predicted the state of H. pylori infection based on the EKSC. Other examiners reviewed all images in the PACS (Kim YJ, >500 diagnostic endoscopies per year with 7 years of experience; Jo $\mathrm{IH}$, $>500$ diagnostic endoscopies per year with 3 years of experience).

In case of disagreement with two examiners, they were excluded in the study. Additionally, we evaluated specific endoscopic findings that might be correlated with $H . p y^{-}$ lori infection state, including mucosal swelling, sticky mucus, red streak, hematin, reflux esophagitis, and duodenitis. Figs. 1-3 show clinical photos of endoscopic findings.

1) Mucosal swelling (soft, thick, and distended mucosa) is shown in Fig. 1A.

2) Sticky mucus (mucus visualized at non-atrophic area in greater curvature of the gastric body) is shown in Fig. $1 \mathrm{~B}$.

3) Red streak (longitudinal red streaks at gastric body and antrum) is shown in Fig. 2A.

4) Hematin (red or dark ecchymosis spots or flecks) is shown in Fig. 2B.

5) Reflux esophagitis (patchy, striated, or circular epithelial defects in the mucosa in the distal esophagus) is shown in Fig. 2C. We also included erythema, blurred junction between squamous epithelium and columnar epithelium, and increased vascular marking in the distal esophagus as minor changes.
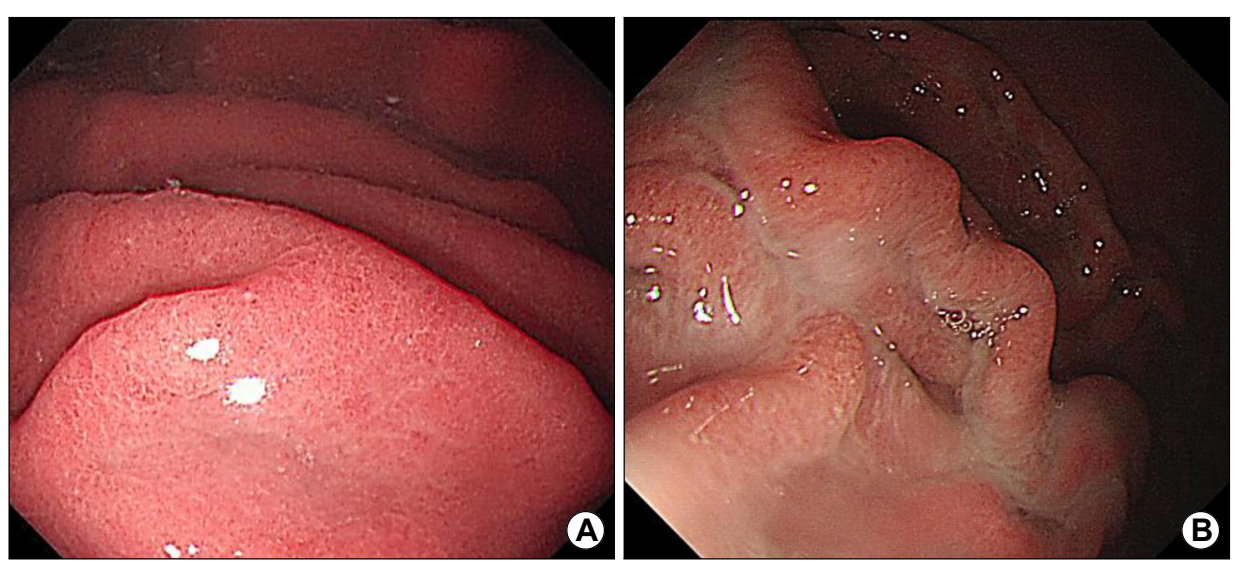

Fig. 1. Specific endoscopic findings correlated with $H$. pylori infection state. Mucosal swelling (A) and sticky mucus (B). H. pylori, Helicobacter pylori. 
6) Duodenitis (erythematous or exudative change with erosions in the bulb or post bulbar area) is shown in Fig. 2D.

7) A normal RAC pattern (small, round pits surrounded by honeycomb-like subepithelial capillary networks [SECN]) is shown in Fig. 3A.

8) An abnormal pattern correlating with $H$. pylori infection (enlarged or prolonged pits with increased density of irregular vessels) is shown in Fig. 3B.

Mucosal swelling and sticky mucus were positively correlated whereas red streak and hematin were negatively correlated with $H$. pylori infection. In a previous study. ${ }^{11}$ These specific endoscopic findings showed relatively high sensitivity, specificity, and response rate for endoscopic examiners. Atrophy, intestinal metaplasia, enlarged folds, nodularity, and RAC pattern were already included in the
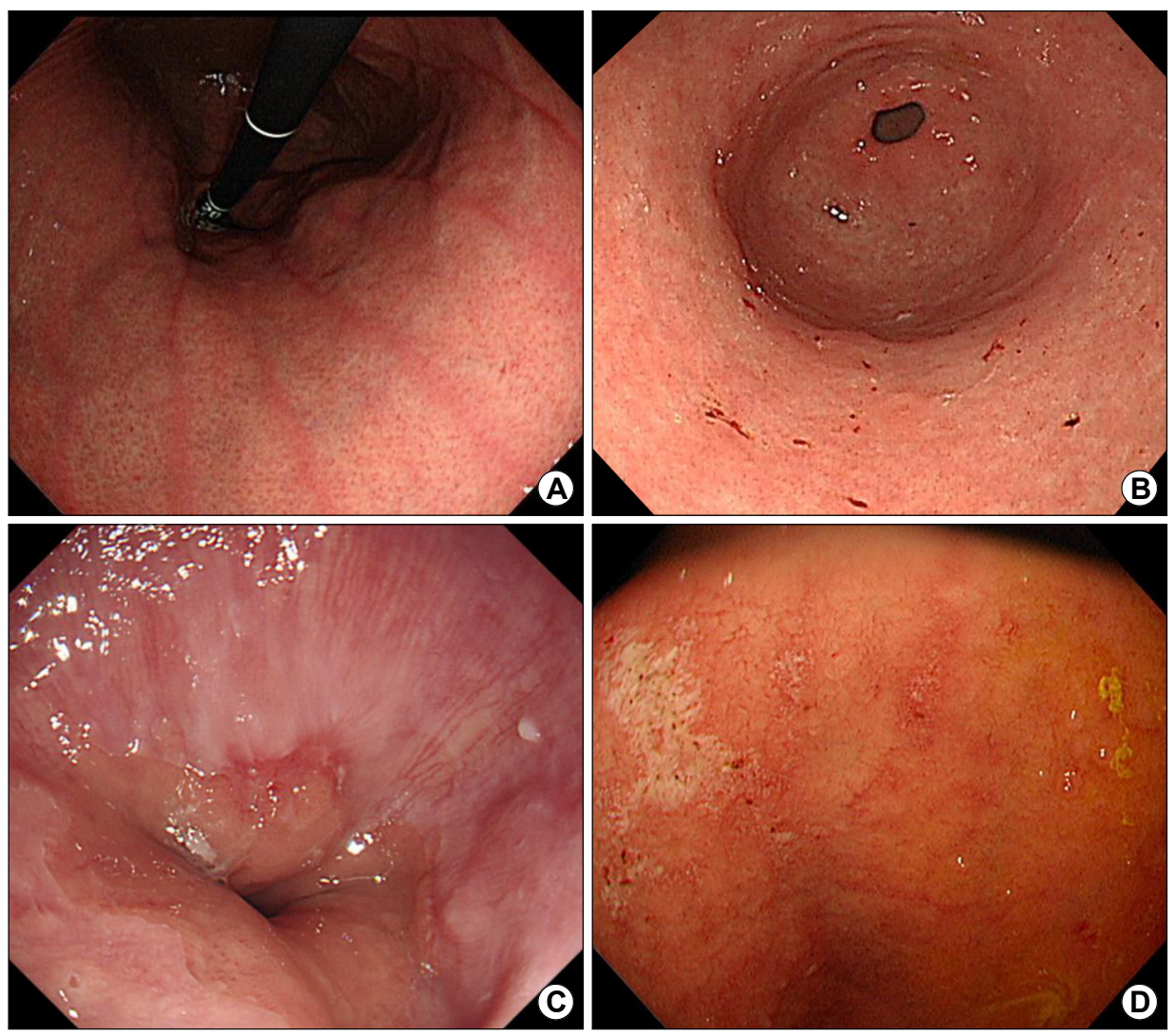

Fig. 2. Specific endoscopic findings, which are not correlated with $H$. pylori infection state. Red streak (A), hematin (B), reflux esophagitis (C), and duodenitis (D). H. pylori, Helicobacter pylori.
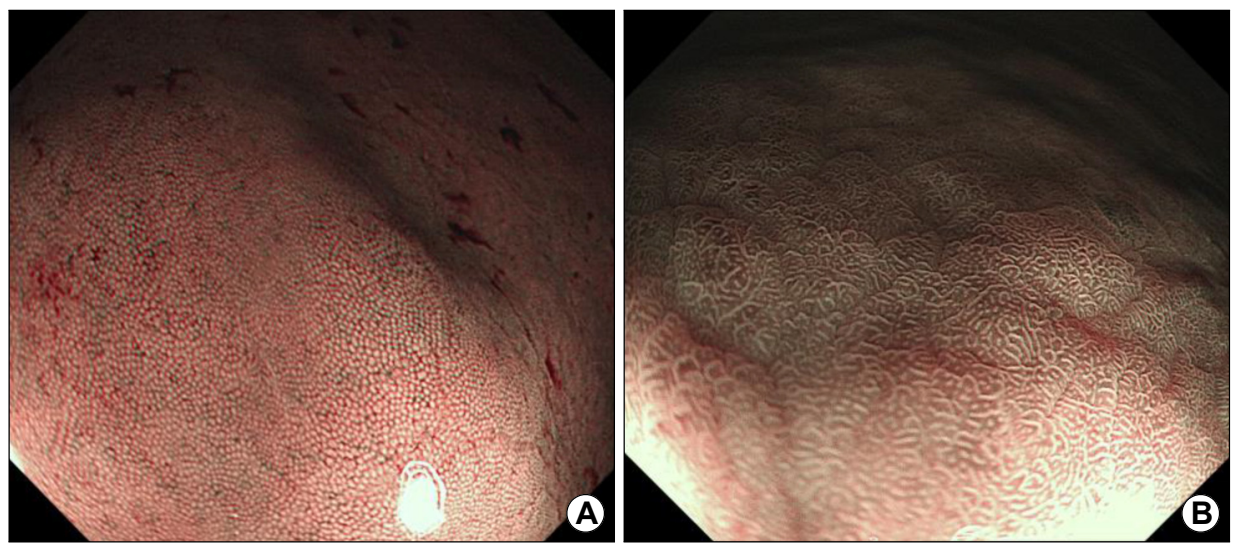

Fig. 3. Narrow band imaging with magnifying endoscopy. The normal regular arrangement of collecting venules (RAC) pattern (A) and the abnormal pattern correlating with $H$. pylori infection (B) are shown. H. pylori, Helicobacter pylori. 
Kyoto scoring system. In this study, we did not consider other specific endoscopic findings such as fundic gland polyp, spotty redness, patch erythema, map like redness, or hyperplastic polyp because they had low response rates despite high sensitivities. As for reflux esophagitis and duodenitis, previous studies have shown equivocal results for their correlations with $H$. pylori infection. ${ }^{18,19}$

\section{Statistical analysis}

All data were recorded on standard forms and analyzed with a computer. Student's $t$-test was used to compare continuous variables between the two groups. Differences between dichotomous variables were evaluated with chisquare test. All statistical analyses were performed using SPSS package software version 25.0 (SPSS Inc., chicago, IL, USA). $P$-values less than 0.05 were considered significant.

\section{RESULTS}

Overall, 1,179 patients were evaluated and 343 patients were excluded due to incomplete evaluation, equivocal results, or disagreement between examiners. A total of 836 patients were enrolled in this study. Their mean age was $63.1 \pm 12.0$ years (range, 18 87). The male:female ratio was 1.17:1 (451 males and 385 females). When they were divided into two groups based on previous history of $H$. pylori eradication, 485 were $H$. pylori naive and 351 had a successful eradication history (Fig. 4).

\section{Diagnostic accuracy by EKSS}

EKSS was anticipated to predict $H$. pylori infection state. When cutoff value was 3 points of the EKSS, sensitivity and specificity were 88.8\% (95\% CI, 84.4 92.3\%) and $81.2 \%$ (95\% CI, 75.4 86.2\%), respectively. The area under the curve (AUC) was 0.90 (95\% CI, 0.87 0.93) for H. pylori naïve patients. In patients with successful eradication history, EKSS had a sensitivity of $85.7 \%$ (95\% CI, 59.5 98.3\%) and a specificity of 68.5\% (95\% CI, 55.9 79.5\%) for $H$. pylori re-infection. The AUC was 0.83 (95\% CI, 0.75 0.92) (Fig. 5A).

When only RAC score (cutoff value: 1 point) was used to predict $H$. pylori infection state, its sensitivity, specificity, and AUC were 91.0\% (95\% CI, 86.9 94.2\%), 82.1\% (95\% CI, 76.4 87.0\%), and 0.88 (95\% CI, 0.85 0.91), respectively, for $H$. pylori naïve patients and 93.3\% (95\% CI, 68.1 99.8\%), 71.4\% (95\% CI, 66.3 76.2\%), and 0.85 (95\%

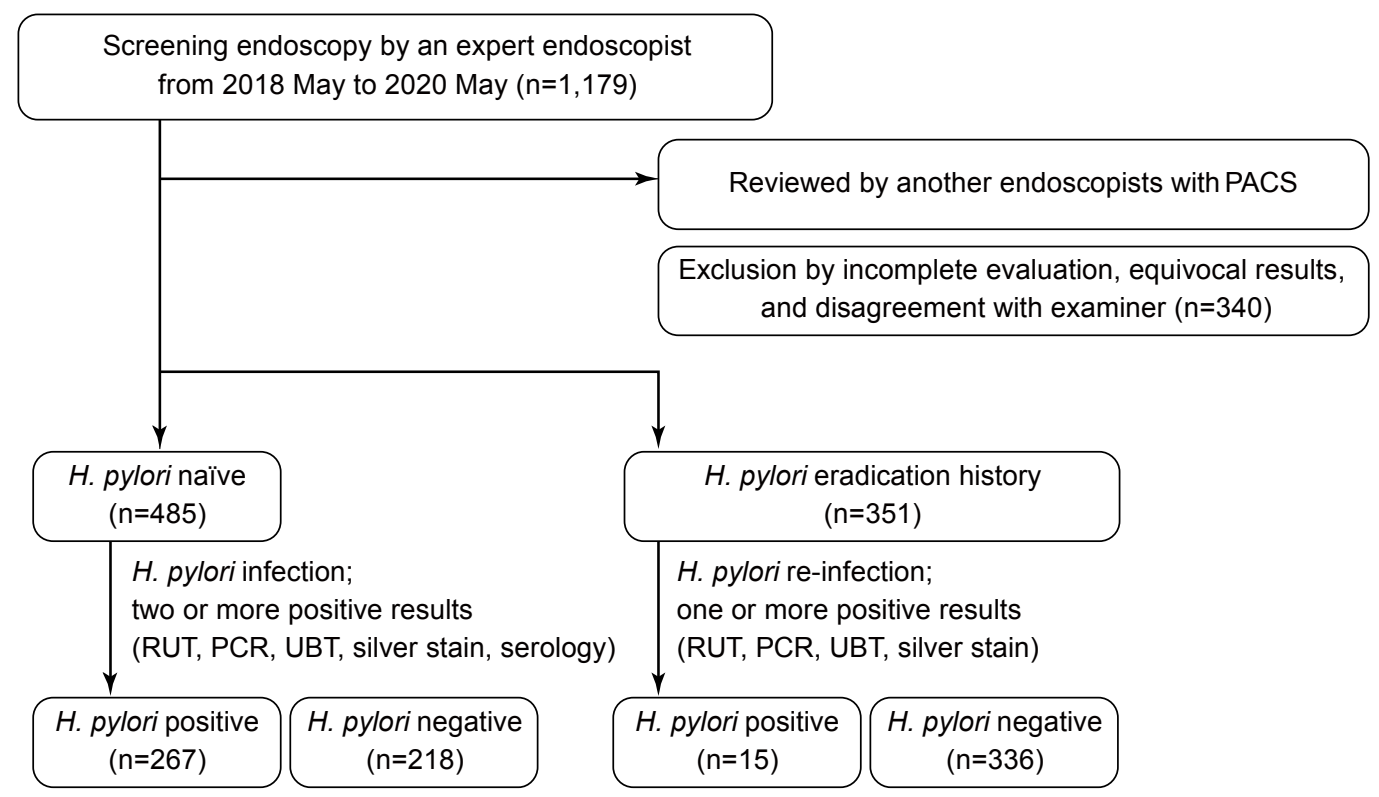

Fig. 4. Flow chart showing patient enrollment in this study. A total of 836 patients were enrolled, including $H$. pylori naïve patients $(n=485)$ and those with a history of $H$. pylori eradication $(\mathrm{n}=351)$. PACS, picture archiving and communicating system; RUT, rapid urease test; PCR, polymerase chain reaction; UBT, urea breath test; H. pylori, Helicobacter pylori. 
CI, 0.77 0.93), respectively, for patients with a successful eradication history (Fig. 5B).

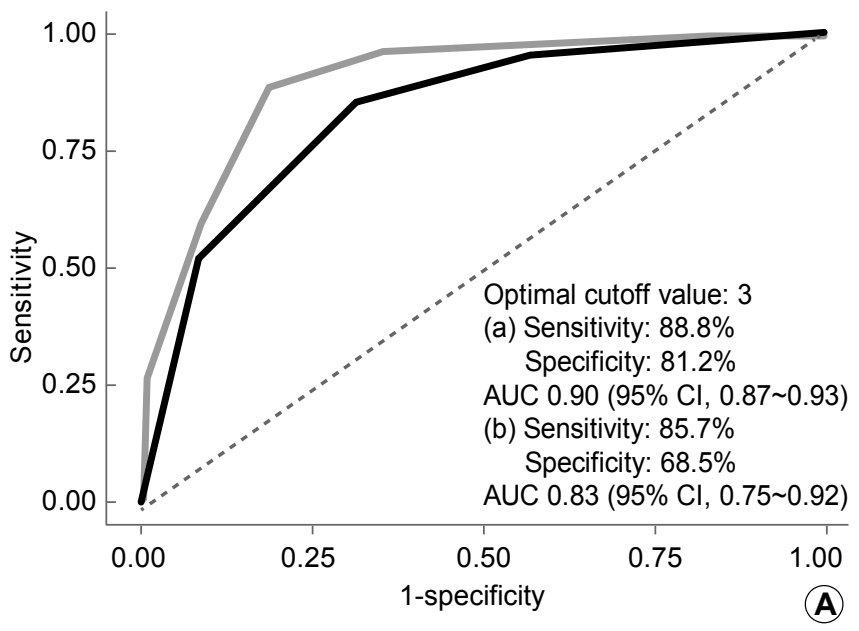

\section{Diagnostic accuracy in patients with open type atrophy and/or intestinal metaplasia}

When patients with open type atrophy (2 points) and/or intestinal metaplasia (1 point) were selected, with a cutoff

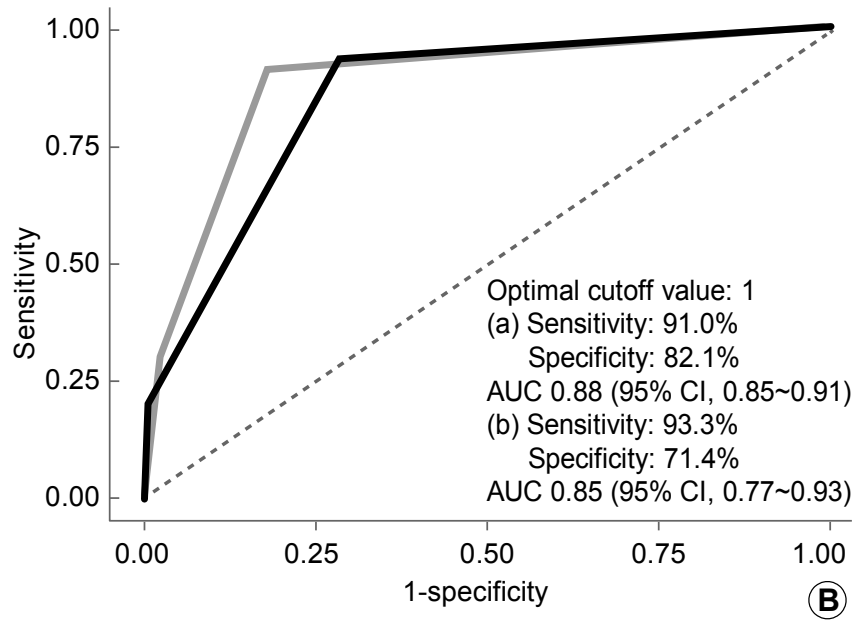

Fig. 5. Diagnostic accuracy of the endoscopic Kyoto scoring system (A) and diffuse redness (B). Both systems provided excellent diagnostic results for predicting $H$. pylori infection. The gray line (a) for patients with $H$. pylori naïve patients; black line (b) for those with a history of $H$. pylori eradication. H. pylori, Helicobacter pylori; AUC, area under the curve; CI, confidence interval.

Table 1. Characteristics of H. pylori Naïve Patients Enrolled in the Study

\begin{tabular}{|c|c|c|c|}
\hline & H. pylori-positive & H. pylori-negative & $P$-value \\
\hline H. pylori naïve $(\mathrm{n}=485)$ & 267 & 218 & \\
\hline Sex (male) & 139 & 100 & \\
\hline Age & $62.70 \pm 14.65$ & $60.66 \pm 11.65$ & 0.08 \\
\hline Diagnosis & & & 0.60 \\
\hline Gastric ulcer & 38 & 26 & \\
\hline Duodenal ulcer & 12 & 8 & \\
\hline Chronic atrophic gastritis & 185 & 154 & \\
\hline Gastric epithelial neoplasia & 32 & 30 & \\
\hline Atrophy & & & $<0.01$ \\
\hline (closed type) & 126 & 105 & \\
\hline (open type) & 139 & 41 & \\
\hline Intestinal metaplasia & 37 & 16 & 0.02 \\
\hline Kyoto score & $3.75 \pm 0.46$ & $1.39 \pm 0.59$ & $<0.01$ \\
\hline Auto-antibody & 5 & 6 & 0.52 \\
\hline \multicolumn{4}{|l|}{ Special endoscopic finding } \\
\hline Mucosal swelling & 231 & 46 & $<0.01$ \\
\hline Sticky mucus & 147 & 21 & $<0.01$ \\
\hline Reflux esophagitis & 61 & 99 & $<0.01$ \\
\hline Hemorrhagic spots & 37 & 83 & $<0.01$ \\
\hline Red streak & 17 & 95 & $<0.01$ \\
\hline Duodenitis & 30 & 36 & 0.09 \\
\hline
\end{tabular}

H. pylori, Helicobacter pylori. 
value of 3 points, sensitivity, specificity, and AUC of the EKSS were 100.0\% (95\% CI, 97.4 100.0\%), 24.4\% (95\% CI, $12.4 \sim 0.3 \%$ ), and 0.62 (95\% CI, 0.56 0.69), respectively, in H. pylori naïve patients and 100.0\% (95\% CI, 75.3 100.0\%), $22.4 \%$ (95\% CI, 15.2 31.1\%), and 0.61 (95\% CI, 0.57 0.65), respectively, in patients with a successful eradication history.

When only RAC score (cutoff value: 1 point) was used to predict $H$. pylori infection state in patients with severe atrophy and/or intestinal metaplasia, its sensitivity, specificity, and AUC were 97.9\% (95\% CI, 93.8 99.6\%), 46.3\% (95\% CI, 30.7 62.9\%), and 0.79 (95\% CI, 0.71 0.86), respectively, for $H$. pylori naïve patients and 100.0\% (95\% CI, 75.3 100.0\%), 65.5\% (95\% CI, 56.1 74.1\%), and 0.88 (95\% CI, 0.83 0.94), respectively, for patients with a successful eradication history.

\section{Specific endoscopic findings}

Mucosal swelling and sticky mucus were positively correlated with infection state, whereas reflux esophagitis, red streak, and hematin were negatively correlated with infection state in patients with $H$. pylori naïve or a history of successful eradication (Tables 1, 2). The endoscopic finding of mucosal swelling had a sensitivity of $86.2 \%$ (95\% CI, 81.6 90.0\%), a specificity of $77.6 \%(95 \%$ CI, 73.9 81.0\%), and a positive predictive value (PPV) of $66.2 \%$ (95\% CI, 62.5 69.7\%) for H. pylori infection. Also, sticky mucus was a critical diagnostic clue with a sensitivity of $54.3 \%(95 \% \mathrm{CI}, 48.2 \sim 60.2 \%)$, a specificity of $93.3 \%$ (95\% CI, 90.9 95.3\%), and a PPV of $80.5 \%(95 \% \mathrm{CI}$, 74.8 85.2\%). When we evaluated their diagnostic values in patients with diffuse atrophy and/or intestinal metaplasia, mucosal swelling and sticky mucus could predict $H$. pylori infection state with sensitivity of $70.6 \%(95 \%$ CI, 62.4 $77.9 \%)$ and specificity of $68.9 \%$ (95\% CI, 53.4 81.8\%) for H. pylori naïve patients ( $\mathrm{n}=188)$. Their sensitivity and specificity were 50.0\% (95\% CI, 21.1 78.9\%) and 93.5\% (95\% CI, 88.0 96.9\%), respectively, for patients with a successful eradication history $(\mathrm{n}=151)$.

As for reflux esophagitis, hematin, red streak, and duodenitis, they showed negative correlations with $H$. pylori

Table 2. Characteristics of Enrolled Patients with a History of Successful H. pylori Eradication

\begin{tabular}{|c|c|c|c|}
\hline & H. pylori-positive & H. pylori-negative & $P$-value \\
\hline H. pylori eradication history $(\mathrm{n}=351)$ & 15 & 336 & \\
\hline Sex (male) & 10 & 202 & \\
\hline Age & $65.98 \pm 8.47$ & $64.74 \pm 9.98$ & 0.61 \\
\hline Diagnosis & & & 0.47 \\
\hline Gastric ulcer & 3 & 48 & \\
\hline Duodenal ulcer & 1 & 25 & \\
\hline Chronic atrophic gastritis & 3 & 119 & \\
\hline Gastric epithelial neoplasia & 8 & 144 & \\
\hline Atrophy & & & 0.02 \\
\hline (closed type) & 5 & 185 & \\
\hline (open type) & 10 & 129 & \\
\hline Intestinal metaplasia & 2 & 81 & 0.34 \\
\hline Kyoto score & $3.50 \pm 0.52$ & $2.08 \pm 0.47$ & $<0.01$ \\
\hline Auto-antibody & 2 & 7 & $<0.01$ \\
\hline \multicolumn{4}{|l|}{ Special endoscopic finding } \\
\hline Mucosal swelling & 12 & 78 & $<0.01$ \\
\hline Sticky mucus & 6 & 16 & $<0.01$ \\
\hline Reflux esophagitis & 2 & 169 & $<0.01$ \\
\hline Hemorrhagic spots & 3 & 186 & 0.03 \\
\hline Red streak & 0 & 80 & $<0.01$ \\
\hline Duodenitis & 0 & 78 & $<0.01$ \\
\hline
\end{tabular}

H. pylori, Helicobacter pylori. 
infection in all subjects. There was a significant difference in negative predictive value (NPV) between $H$. pylori na- ïve patients and those with a history of successful eradication. Estimated NPV for patients with a history of

Table 3. Diagnostic Parameters of Four Endoscopic Findings (Reflux Esophagitis, Hematin, Red Streak, and Duodenitis) for Predicting H. pylori Infection

\begin{tabular}{|c|c|c|c|c|c|c|c|c|}
\hline & \multicolumn{4}{|c|}{ H. pylori naïve } & \multicolumn{4}{|c|}{ History of $H$. pylori eradication } \\
\hline & $\begin{array}{c}\text { Reflux } \\
\text { esophagitis }\end{array}$ & Hematin & Red streak & Duodenitis & $\begin{array}{c}\text { Reflux } \\
\text { esophagitis }\end{array}$ & Hematin & Red streak & Duodenitis \\
\hline Sensitivity & $\begin{array}{c}0.23 \\
(0.18,0.28)\end{array}$ & $\begin{array}{c}0.14 \\
(0.10,0.19)\end{array}$ & $\begin{array}{c}0.06 \\
(0.04,0.10)\end{array}$ & $\begin{array}{c}0.11 \\
(0.08,0.16)\end{array}$ & $\begin{array}{c}0.20 \\
(0.04,0.48)\end{array}$ & $\begin{array}{c}0.20 \\
(0.04,0.48)\end{array}$ & $\begin{array}{c}0.00 \\
(0.00,0.22)\end{array}$ & $\begin{array}{c}0.00 \\
(0.00,0.22)\end{array}$ \\
\hline Specificity & $\begin{array}{c}0.55 \\
(0.48,0.61)\end{array}$ & $\begin{array}{c}0.62 \\
(0.55,0.68)\end{array}$ & $\begin{array}{c}0.56 \\
(0.50,0.63)\end{array}$ & $\begin{array}{c}0.83 \\
(0.78,0.88)\end{array}$ & $\begin{array}{c}0.50 \\
(0.44,0.55)\end{array}$ & $\begin{array}{c}0.45 \\
(0.39,0.50)\end{array}$ & $\begin{array}{c}0.76 \\
(0.71,0.81)\end{array}$ & $\begin{array}{c}0.77 \\
(0.72,0.81)\end{array}$ \\
\hline PPV & $\begin{array}{c}0.38 \\
(0.31,0.46)\end{array}$ & $\begin{array}{c}0.31 \\
(0.23,0.40)\end{array}$ & $\begin{array}{c}0.15 \\
(0.09,0.23)\end{array}$ & $\begin{array}{c}0.45 \\
(0.33,0.58)\end{array}$ & $\begin{array}{c}0.02 \\
(0.00,0.05)\end{array}$ & $\begin{array}{c}0.02 \\
(0.00,0.05)\end{array}$ & $\begin{array}{c}0.00 \\
(0.00,0.05)\end{array}$ & $\begin{array}{c}0.00 \\
(0.00,0.05)\end{array}$ \\
\hline NPV & $\begin{array}{c}0.37 \\
(0.31,0.42)\end{array}$ & $\begin{array}{c}0.37 \\
(0.32,0.42)\end{array}$ & $\begin{array}{c}0.33 \\
(0.28,0.38)\end{array}$ & $\begin{array}{c}0.43 \\
(0.39,0.48)\end{array}$ & $\begin{array}{c}0.93 \\
(0.89,0.96)\end{array}$ & $\begin{array}{c}0.93 \\
(0.87,0.96)\end{array}$ & $\begin{array}{c}0.94 \\
(0.91,0.97)\end{array}$ & $\begin{array}{c}0.95 \\
(0.91,0.97)\end{array}$ \\
\hline
\end{tabular}

H. pylori, Helicobacter pylori; PPV, positive predictive value; NPV, negative predictive value.

Table 4. Four-item 4-point Scale of Endoscopic Findings (Hematin, Red Streak, Reflux Esophagitis, and Duodenitis) for Predicting H. pylori Infection in H. pylori Naïve Patients (A) and Those with a History of H. pylori Eradication (B)

\begin{tabular}{lrrrrrr}
\hline & \multicolumn{2}{c}{ Cutoff value } & \multirow{2}{*}{ 1 point } & 2 points & \multirow{2}{*}{3 points } & \multirow{2}{*}{4 points } \\
\cline { 2 - 4 } (A) H. pylori naïve patients & 28 & 105 & 133 & 276 & 42 & 7 \\
Scale (+) & 239 & 113 & 352 & 209 & 443 & 478 \\
Scale (-) & 267 & 218 & 485 & 485 & 485 & 485 \\
Total & 15 & 317 & 332 & 215 & 143 & 49 \\
(B) Patients with a history of H. pylori eradication & & & & & & \\
Scale (+) & 0 & 19 & 19 & 136 & 208 & 302 \\
Scale (-) & 15 & 336 & 351 & 351 & 351 & 351 \\
Total & & & & & & \\
\hline
\end{tabular}

H. pylori, Helicobacter pylori.

Table 5. Diagnostic Parameters of a 4-item 4-point Scale of Endoscopic Findings (Hematin, Red Streak, Reflux Esophagitis, and Duodenitis) for Predicting H. pylori Infection in H. pylori Naïve Patients (A) and Those with a History of H. pylori Eradication (B)

\begin{tabular}{lcccc}
\hline & 1 point & 2 points & 3 points & 4 points \\
\hline (A) H. pylori naïve patients & & & & \\
Apparent prevalence & $0.27(0.23,0.32)$ & $0.57(0.52,0.61)$ & $0.09(0.06,0.12)$ & $0.01(0.01,0.03)$ \\
True prevalence & $0.55(0.51,0.60)$ & $0.55(0.51,0.60)$ & $0.55(0.51,0.60)$ & $0.55(0.51,0.60)$ \\
Sensitivity & $0.10(0.07,0.15)$ & $0.42(0.36,0.48)$ & $0.02(0.01,0.04)$ & $0.00(0.00,0.01)$ \\
Specificity & $0.52(0.45,0.59)$ & $0.25(0.19,0.31)$ & $0.83(0.77,0.88)$ & $0.97(0.93,0.99)$ \\
Positive predictive value & $0.21(0.14,0.29)$ & $0.41(0.35,0.47)$ & $0.12(0.04,0.26)$ & $0.00(0.00,0.41)$ \\
Negative predictive value & $0.32(0.27,0.37)$ & $0.26(0.20,0.32)$ & $0.41(0.36,0.46)$ & $0.44(0.40,0.49)$ \\
Positive likelihood ratio & $0.22(0.15,0.32)$ & $0.56(0.47,0.65)$ & $0.11(0.04,0.28)$ & $0.00(0.00,0.00)$ \\
Negative likelihood ratio & $1.73(1.51,1.98)$ & $2.34(1.82,3.02)$ & $1.18(1.11,1.26)$ & $1.03(1.01,1.06)$ \\
(B) Patients with a history of . pylori eradication & & & & \\
Apparent prevalence & $0.95(0.92,0.97)$ & $0.61(0.56,0.66)$ & $0.41(0.36,0.46)$ & $0.14(0.11,0.18)$ \\
True prevalence & $0.04(0.02,0.07)$ & $0.04(0.02,0.07)$ & $0.04(0.02,0.07)$ & $0.04(0.02,0.07)$ \\
Sensitivity & $1.00(0.78 .1 .00)$ & $0.93(0.68,1.00)$ & $0.87(0.60,0.98)$ & $0.60(0.32,0.84)$ \\
Specificity & $0.06(0.03,0.09)$ & $0.40(0.35,0.46)$ & $0.61(0.56,0.67)$ & $0.88(0.84,0.91)$ \\
Positive predictive value & $0.05(0.03,0.07)$ & $0.07(0.04,0.11)$ & $0.09(0.05,0.15)$ & $0.18(0.09,0.32)$ \\
Negative predictive value & $1.00(0.82,1.00)$ & $0.99(0.96,1.00)$ & $0.99(0.97,1.00)$ & $0.98(0.96,0.99)$ \\
Positive likelihood ratio & $1.06(1.03,1.09)$ & $1.56(1.33,1.83)$ & $2.24(1.76,2.85)$ & $5.04(3.04,8.35)$ \\
Negative likelihood ratio & $0.00(0.00,0.00)$ & $0.17(0.02,1.11)$ & $0.22(0.06,0.79)$ & $0.45(0.24,0.84)$ \\
\hline
\end{tabular}

H. pylori, Helicobacter pylori. 
successful eradication was higher than that for $H$. pylori naïve patients (Table 3). When endoscopic duodenitis was observed, it only showed a negative correlation with $H$. pylori infection in patients with a history of successful eradication, with an NPV of 95.0\% (95\% CI, 91.0 96.9\%). When we evaluated discrimination system using the four endoscopic findings mentioned above with a 4-point scale, patients with a history of eradication showed high NPVs (Tables 4, 5).

\section{DISCUSSION}

Increasing antibiotic resistance rate, including clarithromycin resistance that affects $H$. pylori eradication therapy, is a challenge for clinicians. Based on $23 \mathrm{~S}$ ribosomal ribonucleic acid point mutations, the resistance rate for clarithromycin has increased drastically up to $38.5 \%$ in Korea. ${ }^{8}$ The standard triple regimen is no longer suitable as first-line treatment. There is a growing interest in personalized care for patients. Tailored therapy based on antibiotic susceptibility could increase $H$. pylori eradication rate. $^{20,21}$ Therefore, in situ endoscopic diagnosis of $H . p y^{-}$ lori infection could improve the efficacy of eradication therapy. When clues for $H$. pylori infection are observed during endoscopy, an antibiotic resistance test can be performed with a one-step process. Subsequently, clinicians could choose an optimal regimen based on resistance results. Moreover, the strategy of screen-and-treat for $H$. pylori infection would be the most cost-effective in regions with a highly prevalence of gastric cancers, especially Korea.

This is the first study that provides evidence for the usefulness of EKSS for predicting H. pylori state in patients with a history of eradication. EKSS consists of scores for gastric atrophy, intestinal metaplasia, enlarged folds, nodularity, and diffuse redness. Several studies have investigated its diagnostic values since its introduction in 2015. A retrospective study has analyzed $H$. pylori naïve patients whose serum anti-H. pylori antibody has a negative-high titer (defined as 3 9.9 U/mL) and reported that the accuracy of EKSS for H. pylori infection is $89.7 \%$ when it is analyzed with a score of 2 or higher. ${ }^{16}$ Another study has analyzed endoscopic images of $870 \mathrm{H}$. pylori naïve patients and found that the infection rate shows an increasing pattern when the score of EKSS is increased from 0 to 2 or more (up to $82 \%$ ). ${ }^{22}$ Our results revealed that AUC values of ROC curves were favorable (over 0.8) in both $H$. pylori naïve patients and those with a reinfection. However, the optimal criterion of our study was 3 points whereas it was 2 points in aforementioned studies. Results of our study revealed that EKSS as a screening tool was valuable for both $H$. pylori naivve patients and those with a history of eradication. However, EKSS had a lower diagnostic accuracy for those with a re-infection of $H$. pylori compared to that for $H$. pylori naïve patients.

The degree of visibility of RAC pattern at body constituting the EKSS provided excellent diagnostic results for predicting $H$. pylori infection, comparable to that of the EKSS. RAC pattern of gastric mucosa is closely related to H. pylori infection status in previous studies. ${ }^{23,24}$ According to our data, RAC score alone could replace EKSS for predicting $H$. pylori infection state. Especially, when a far progressed atrophic change (open type atrophy) is present with or without intestinal metaplasia, EKSS would be 3 points or more, the cutoff level for $H$. pylori infection. In highly prevalent region of $H$. pylori, the above endoscopic findings (open type atrophy and intestinal metaplasia) could be common. Taken together, RAC score alone could be a simpler and better predictor for $\mathrm{H}$. pylori infection state than EKSS. Unfortunately, its specificity was disappointing.

Because of limitations of in-site diagnosis using EKSS or RAC pattern, there would be a considerable need for auxiliary endoscopic findings for more accurate decision. The presence of mucosal swelling and sticky mucus had a relatively high specificity for predicting $H$. pylori infection state, even in patients with diffuse atrophy and/or intestinal metaplasia. Simply, the presence of RAC score, mucosal swelling, and sticky mucus could diagnose $H$. pylori infection. In the same manner, the presence of hematin, red streak, reflux esophagitis, and duodenitis could help decide the $H$. pylori negative state, showing $\mathrm{ex}^{-}$ cellent NPV for patients with a history of $H$. pylori eradication.

Hematin, red streak, and reflux esophagitis on endo- 
scopic findings were negatively related to $H$. pylori infection regardless of the eradication history. Several studies have reported that hematin and reflux esophagitis can suggest an acidic status. ${ }^{25-27}$ Considering that hypoacidity would be slowly progressed during a long-lasting $H$. pylori infection, hematin and reflux esophagitis might have developed after eradicating $H$. pylori infection. ${ }^{28}$ Also, a high diagnostic odds ratio of red streak for $H$. pylori noninfection state has been reported. ${ }^{29}$ Another interesting finding was that the presence of duodenitis showed remarkable NPV. The relationship between duodenitis and H. pylori infection state remains controversial.

Different from our results, a previous report has suggested that gastric metaplasia of duodenal mucosa could be partially affected by $H$. pylori infection. ${ }^{18}$ Duodenitis is more common in the $H$. pylori positive group than in the H. pylori negative group in high prevalence regions. ${ }^{30}$ Lower $H$. pylori prevalence and higher duodenitis prevalence have been observed in inflammatory bowel disease patients than in the general population. ${ }^{31}$ Surprisingly, our results showed that duodenitis was negatively correlated with $H$. pylori infection, especially in patients with a history of successful eradication. It was unlikely that the presence of endoscopic duodenitis was suspected as $H$. pylori infection in H. pylori naïve patients. These opposite results could be explained by differences in $H$. pylori prevalence. In addition, a relatively long duration of infection might have influenced these differences. Moreover, the diagnosis of negative infection of $H$. pylori was made based on two or more negative results of RUT, PCR, UBT, histologic evidence by silver stain, and serologic test. Our study had a well-designed control group, whereas most of previous studies did not include a control group. On the other hand, in cases of $H$. pylori-positive gastritis, the suppressive effect induced by inflammatory cells on parietal cells can reduce gastric acid secretion. After the eradication of $H$. pylori, endoscopic duodenitis could result in the recovery of gastric acid secretion.

Our study had several strong points compared with previous studies. To the best of our knowledge, few studies have evaluated the diagnostic performance of EKSS, especially those comparing two different populations according to the presence of previous $H$. pylori eradication treatment. Because the definition of $H$. pylori infection state was strict in this study, the robustness of $H$. pylori diagnosis was kept during the study. All endoscopic images of enrolled patients were examined by one expert and cross-validated independently by two endoscopy specialists. These might have eliminated inter-observer variation and minimized the intervening of other observational bias. Nevertheless, this study has several limitations. First, all results of this study were derived from opinions of only one expert on endoscopic imaging, making it difficult to rule out subjectivity. The diagnostic performance of EKSS might be different depending on the experience of each endoscopist. Second, the endoscopic device used was derived of a single maker (Olympus Corporation, Tokyo, Japan). Results could be different if different equipment are used. Narrow band imaging (NBI) is a patented endoscopic light technology. RAC pattern should be examined by magnifying endoscopy with NBI. Lastly, this study was performed in Korea where the prevalence of $H$. pylori infection was high. In a region with a low prevalence of $H$. pylori infection, the usefulness of EKSS remains unclear.

In conclusion, in situ diagnostic performance of EKSS for predicting $H$. pylori infection was excellent for both H. pylori naïve patients and those with a history of successful eradication. Also, RAC pattern at body could replace EKSS. Although efficacies of EKSS and RAC pattern for predicting $H$. pylori infection were decreasing in patients with open type atrophy and/or intestinal metaplasia, supportive endoscopic findings (mucosal swelling, sticky mucus, reflux esophagitis, hematin, red streak, and duodenitis) could be useful for in situ diagnosis of $H$. $p y^{-}$ lori infection. Especially, mucosal swelling and sticky mucus had relatively high positive predictive values for $H$. pylori infection in naïve patients, whereas reflux esophagitis, hematin, red streak, and duodenitis had high negative predictive values in those with a history of $H$. pylori eradication.

\section{CONFLICT OF INTEREST}

No potential conflict of interest relevant to this article was reported. 


\section{ORCID}

\author{
Eunsun Lim \\ (iD) https://orcid.org/0000-0002-0900-8848 \\ Ik Hyun Jo \\ (D) https://orcid.org/0000-0002-3433-8662 \\ Yeon-Ji Kim \\ (D) https://orcid.org/0000-0001-9823-577X \\ Woo Chul Chung
}

\section{REFERENCES}

1. El-Zimaity H, Choi WT, Lauwers GY, Riddell R. The differential diagnosis of Helicobacter pylori negative gastritis. Virchows Arch 2018;473:533-550.

2. Uemura N, Okamoto S, Yamamoto S, et al. Helicobacter pylori infection and the development of gastric cancer. N Engl J Med 2001;345:784-789.

3. Stolte M, Bayerdörffer E, Morgner A, et al. Helicobacter and gastric MALT lymphoma. Gut 2002;50:iii19-iii24.

4. Zullo A, Hassan C, Romiti A, et al. Follow-up of intestinal metaplasia in the stomach: when, how and why. World J Gastrointest Oncol 2012;4:30-36.

5. Kim DB, Chung WC. Accuracy of Endoscopic diagnosis of mild atrophic gastritis with Helicobacter pylori infection. Clin Endosc 2018;51:310-312.

6. Díaz P, Valenzuela Valderrama M, Bravo J, Quest AFG. Helicobacter pylori and gastric cancer: adaptive cellular mechanisms involved in disease progression. Front Microbio 2018;9:5.

7. Malfertheiner P, Fry LC, Mönkemüller K. Can gastric cancer be prevented by Helicobacter pylori eradication? Best Pract Res Clin Gastroenterol 2006;20:709-719.

8. Kim YJ, Chung WC. Eradication therapy for Helicobacter pylori with diagnostic test for clarithromycin resistance. Korean J Helicobacter Up Gastrointest Res 2019;19:225-230.

9. Lee SY. Helicobacter pylori infection and the Kyoto classification of gastritis. Korean J Helicobacter Up Gastrointest Res 2019;19:81-87.

10. Kim GH. Endoscopic findings of Kyoto classification of gastritis. Korean J Helicobacter Up Gastrointest Res 2019;19:88-93.

11. Okamura T, Iwaya Y, Kitahara K, Suga T, Tanaka E. Accuracy of endoscopic diagnosis for mild atrophic gastritis infected with Helicobacter pylori. Clin Endosc 2018;51:362-367.

12. Kim BC, Song MA, Kwon SH, Kim MJ. Assessment of gastric acidity by conventional endoscopy with serological gastric markers. Gastroenterology Res 2018;11:112-123.

13. Sugano K, Tack J, Kuipers EJ, et al. Kyoto global consensus report on Helicobacter pylori gastritis. Gut 2015;64:1353-1367.

14. Lee DH. Kyoto classification of gastritis and gastric cancer. Korean J Helicobacter Up Gastrointest Res 2019;19:94-98.

15. Nakmaura Y, Somary U. Exploring the potential of endoscopic examinations using the Kyoto scoring system for risk evaluation of gastric carcinoma in patients with gastritis. Int J Oncol Res

2017;1:001.

16. Toyoshima O, Nishizawa T, Arita M, et al. Helicobacter pylori infection in subjects negative for high titer serum antibody. World J Gastroenterol 2018;24:1419-1428.

17. Watanabe K, Nagata N, Shimbo T, et al. Accuracy of endoscopic diagnosis of Helicobacter pylori infection according to level of endoscopic experience and the effect of training. BMC Gastroenterol 2013;13:128.

18. Khulusi S, Badve S, Patel P, et al. Pathogenesis of gastric metaplasia of the human duodenum: role of Helicobacter pylori, gastric acid, and ulceration. Gastroenterology 1996;110:452458.

19. Scida S, Russo M, Miraglia C, et al. Relationship between Helicobacter pylori infection and GERD. Acta Biomed 2018; 89(8-S):40-43.

20. Hu Y, Zhu Y, Lu NH. Novel and effective therapeutic regimens for Helicobacter pylori in an era of increasing antibiotic resistance. Front Cell Infect Microbiol 2017;7:168.

21. Sugimoto M, Furuta T. Efficacy of tailored Helicobacter pylori eradication therapy based on antibiotic susceptibility and CYP2C19 genotype. World J Gastroenterol 2014;20:6400-6411.

22. Nishizawa T, Sakitani K, Suzuki H, et al. A combination of serum anti-Helicobacter pylori antibody titer and Kyoto classification score could provide a more accurate diagnosis of $H$ pylori. United European Gastroenterol J 2019;7:343-348.

23. Ono S, Dohi O, Yagi N, et al. Accuracies of endoscopic diagnosis of Helicobacter pylori-gastritis: multicenter prospective study using white light imaging and linked color imaging. Digestion 2020;101:624-630.

24. Garcés-Durán R, García-Rodríguez A, Córdova H, et al. Association between a regular arrangement of collecting venules and absence of Helicobacter pylori infection in a european population. Gastrointest Endosc 2019;90:461-466.

25. Hatta W, Iijima K, Koike T, et al. Endoscopic findings for predicting gastric acid secretion status. Dig Endosc 2015;27:582-589.

26. Iwakiri K, Kawami N. Pathophysiology and treatment of reflux esophagitis. Nihon Rinsho 2015;73:1098-1102.

27. Di Mario F, Goni E. Gastric acid secretion: changes during a century. Best Pract Res Clin Gastroenterol 2014;28:953-965.

28. Waldum HL, Kleveland PM, Sørdal ØF. Helicobacter pylori and gastric acid: an intimate and reciprocal relationship. Therap Adv Gastroenterol 2016;9:836-844.

29. Yoshii S, Mabe K, Watano K, et al. Validity of endoscopic features for the diagnosis of Helicobacter pylori infection status based on the Kyoto classification of gastritis. Dig Endosc 2020; 32:74-83.

30. Sahin A, Cihangiroglu G, Bilgic Y, Calhan T, Cengiz M. Is duodenal biopsy appropriate in areas endemic for Helicobacter pylori? North Clin Istanb 2017;4:13-21.

31. Han Y, Jung HK, Chang JY, et al. Identification of distinctive clinical significance in hospitalized patients with endoscopic duodenal mucosal lesions. Korean J Intern Med 2017;32:827-835. 